\title{
Analysis of seismic noise to check the mechanical isolation of a medical device
}

\author{
Paola Cusano $^{1,{ }^{\star}}$, Antonella Bobbio ${ }^{1}$, Gaetano Festa $^{2}$, Simona Petrosino $^{1}$, Sara Rombetto ${ }^{3}$ \\ ${ }^{1}$ Istituto Nazionale di Geofisica e Vulcanologia, sezione di Napoli, Osservatorio Vesuviano, Naples, Italy \\ ${ }^{2}$ Università degli Studi di Napoli «Federico II», Dipartimento di Scienze Fisiche, Naples, Italy \\ ${ }^{3}$ Istituto di Cibernetica "Eduardo Caianiello», Consiglio Nazionale delle Ricerche, Naples, Italy
}

\author{
Article history \\ Received July 15, 2010; accepted January 31, 2011. \\ Subject classification: \\ Seismology, Ground motion, Seismic noise, Noise acquisition, Anthropogenic sources, Spectral ratio analyses, Magnetoencephalography.
}

\section{ABSTRACT}

We have investigated the mechanical response of a magnetically shielded room that hosts a magnetoencephalography system that is subject to external vibrations. This is a superconducting quantum interference device, which are the most sensitive sensors for magnetic flux variations. When the magnetoencephalography operates with people inside the room, the spectrum of the flux of the magnetic field shows anomalous peaks at several frequencies between $1 \mathrm{~Hz}$ and $20 \mathrm{~Hz}$, independent of the experiment that is being run. As the variations in the flux of the magnetic field through the sensors might not only be related to the electrical currents circulating inside the brain, but also to non-damped mechanical oscillations of the room, we installed seismic instrumentation to measure the effective motion inside the room and to compare it to the external motion. For this analysis, we recorded the ambient seismic noise at two very close stations, one inside the magnetically shielded room, the other one outside in the room in which the magnetically shielded room is itself located. Data were collected over four days, including a week-end, to study the response of the magnetically shielded room subjected to different energy levels of external vibrations. The root mean square, Fourier spectra and power spectral density show significant differences between the signal recorded inside and outside the magnetically shielded room, with several anomalous peaks in the frequency band of $1 \mathrm{~Hz}$ to $20 \mathrm{~Hz}$. The normalized spectral quantities (horizontal to vertical spectral ratio, and ratio between the internal and external spectra) show large amplification at several frequencies, reaching in some cases one order of magnitude. We concluded that the magnetically shielded room does not dampen the external vibrations, but it instead appears to amplify these across a broad frequency range.

\section{Introduction}

The first attempts to record electrical signals generated by the human brain were performed at the end of the $1960 \mathrm{~s}$ with the Cohen experiments [1968]. Two years later, superconducting quantum interference device (SQUID) sensors were used to record electrical signals from the heart, and then the alpha rhythms generated by the brain [Cohen 1972]. Since then, several improvements have been made to locate the sources of this brain activity, and it is now possible to have simultaneous images of the entire brain [Williamson and Kauffman 1990]. This has allowed further investigations into Alzheimer's and Parkinson's diseases, to better understand the mechanisms of the brain cognitive functions, and finally to provide pre-surgical analysis.

Specifically, magnetoencephalography (MEG) is an imaging technique that is used to measure the magnetic fields produced by the electrical activity in the brain via extremely sensitive devices, such as SQUIDs [Hamalainen et al. 1993]. As an aspect of electroencephalography, MEG is a non-invasive human brain-imaging tool that provides submillisecond temporal accuracy to help to unravel the dynamics of cortical function. MEG directly reflects the electrical currents in neurons.

A multichannel SQUID system for MEG measurements is installed in Naples, in the "Villa Russo SpA» Hospital. The SQUID array is packed around an helmet surface, and it consists of 163 integrated SQUID magnetometers. This system [Rombetto 2009] is designed for high sensitivity measurements of the brain magnetic field, which is extremely weak, with a typical magnitude of the order of few picoTesla. For this reason, extremely sensitive magnetic field sensors are needed, with suppression of the background magnetic noise, the amplitude of which is a few microTesla, so as to accurately retrieve the brain magnetic field. Additionally, MEG systems have to be mechanically isolated, to reduce disturbances in flux evaluation associated with ambient seismic noise. Hence, this MEG system is located inside a magnetic shielded room (MSR; Figure 1) that designed to uncouple the system from external motion.

In the operational phase of the SQUID, anomalous peaks were observed in the spectrum of the flux of the magnetic 


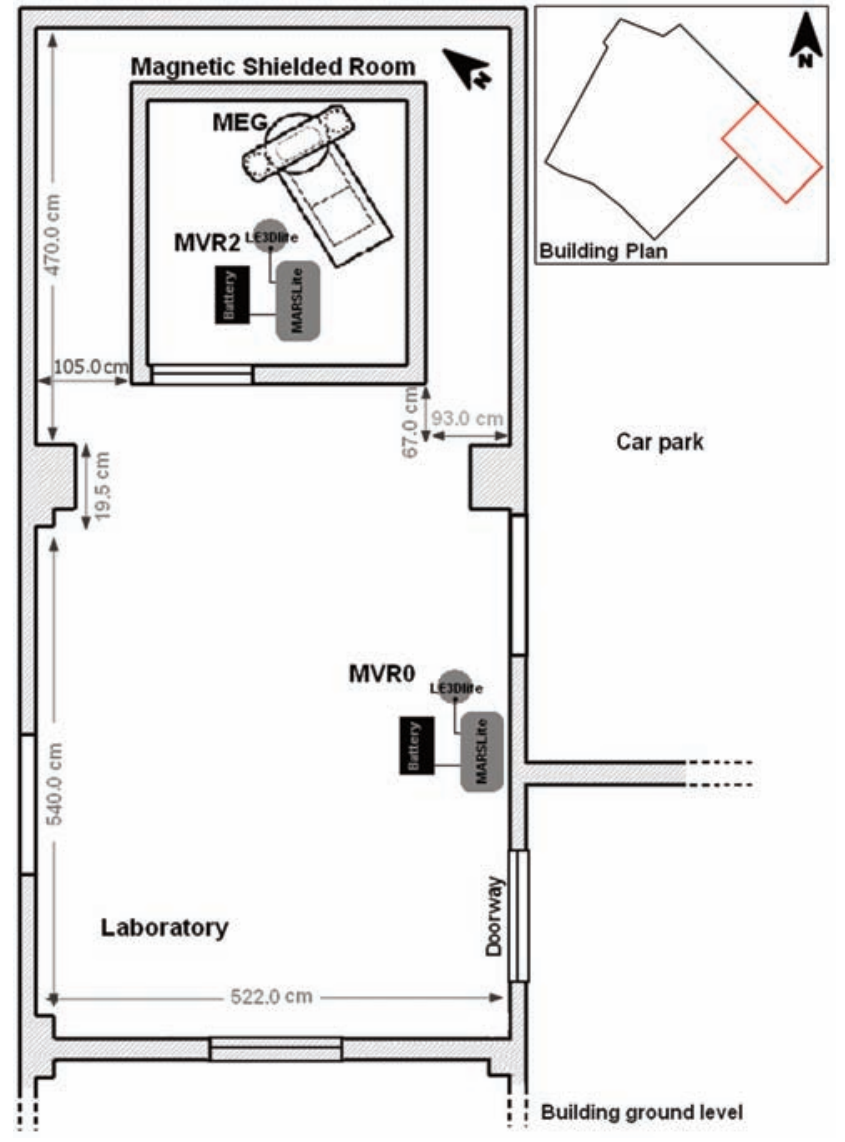

Figure 1. Top right: Building plan of the south-eastern sector of the Villa Russo Hospital complex (Naples, Italy), where the MEG system is hosted. Red line, the two rooms where the MEG system and the two seismic stations, MVR0 and MVR2, are installed. Left: Plan of these two rooms. Top room: The MSR setting, with station MVR2 at the centre, with the MEG system at the top right. This is composed of a couch where a patient lies, a fibreglass dewar with a helmet-shaped bottom, the SQUID sensor array housed around the helmet, and a mechanical support that places the helmet over the had of the patient. Bottom room: The laboratory where the magnetometric data are analyzed, with the location of station MVR0 shown.
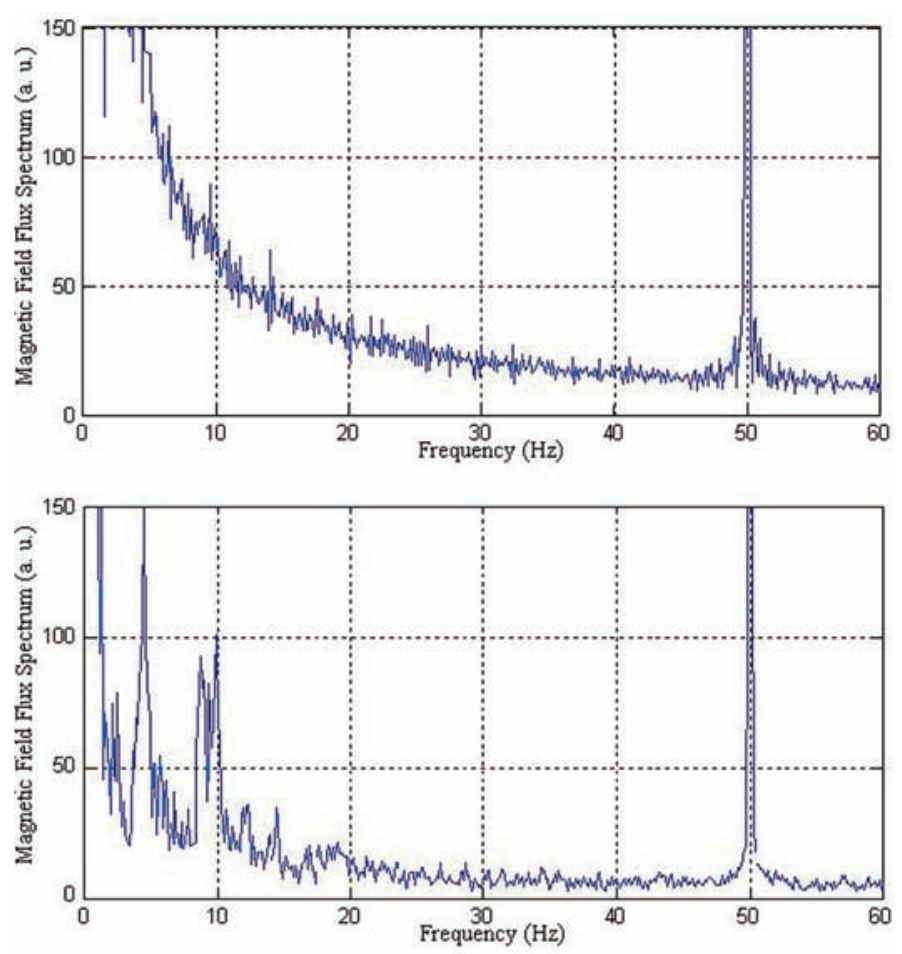

field at several frequencies between $1 \mathrm{~Hz}$ and $20 \mathrm{~Hz}$ (Figure 2). These are likely to be associated with nondamped oscillations of the MSR. Hence, we investigated the mechanical interactions between the soil and the MSR by the use of the ambient seismic noise. This represents an appealing low-cost, fast and non-invasive tool for the investigation of elastic responses of structures to seismic vibrations. At low frequencies $(f<1 \mathrm{~Hz})$, the ambient seismic noise is mostly generated by marine waves breaking along the coast, by the tides, and by the daily temperature variations. At higher frequencies $(f<1 \mathrm{~Hz})$, the ambient noise arises from human activities (e.g., factories, traffic) and meteorological agents (e.g., rainfall, wind). The noise is found to be energetic enough in a broad frequency range to be recorded at levels significantly greater than the electronic disturbance (by both short-period and broad-band seismographs). Hence, the ambient seismic noise is well suited for the evaluation of elastic responses of soils and structures.

\section{Set-up of the experiment}

With the aim of measuring the effective motion inside the MSR in relation to the external motion, we monitored and recorded the high-frequency seismic noise in Villa Russo Hospital.

The instrumentation consisted of two portable seismic stations, MVR0 and MVR2, from the INGV-OV (Istituto Nazionale di Geofisica e Vulcanologia Osservatorio Vesuviano) Mobile Seismic Network. Each station comprised a MARSlite Lennartz datalogger, equipped with a GPS receiver, a 3-component $1-\mathrm{Hz}$ LE3Dlite seismometer (with flat velocity response in the $1-80 \mathrm{~Hz}$ frequency band), and a power supply. The sampling rate was set at 125 sps and the scale at $32 \mu \mathrm{V} /$ count. In these experimental measures, we found that the instrumental noise relative to the MARSlite stations has a maximum excursion of $\pm 50 \mu \mathrm{V}$, which represents a fraction of between 0.01 and 0.001 of the usual level of cultural noise in the urban area of Naples. Data were acquired in the binary GSE format [Group of Scientific Experts 1995] and logged in continuous mode on the DP-PCM2 5 GB PC card. Finally, the data were transferred on a PC hard-disk and converted to SAC format (http://www.llnl.gov/sac/).

The building hosting the MEG device (Figure 1, top right) is located in the south-eastern sector of the hospital. Two rooms on the ground floor are dedicated to the

Figure 2 (left). Examples of the magnetic field flux spectra as functions of the frequency, as recorded by magnetometer $n^{\circ} 16$ of the Villa Russo SQUIDS. Top: data recorded on February 4, 2010, at 12:33. Bottom: data recorded on February 4, 2010, at 10:03. The spectra refer to measurements in the "empty" situation (no-one inside the MSR), showing the ideal "empty" condition where the spectrum is characterized by an absence of peaks, except for the peak at $50 \mathrm{~Hz}$ that is associated with the electrical power supply (top), and the anomalous peaks observed between $1 \mathrm{~Hz}$ and $20 \mathrm{~Hz}$ (bottom). 


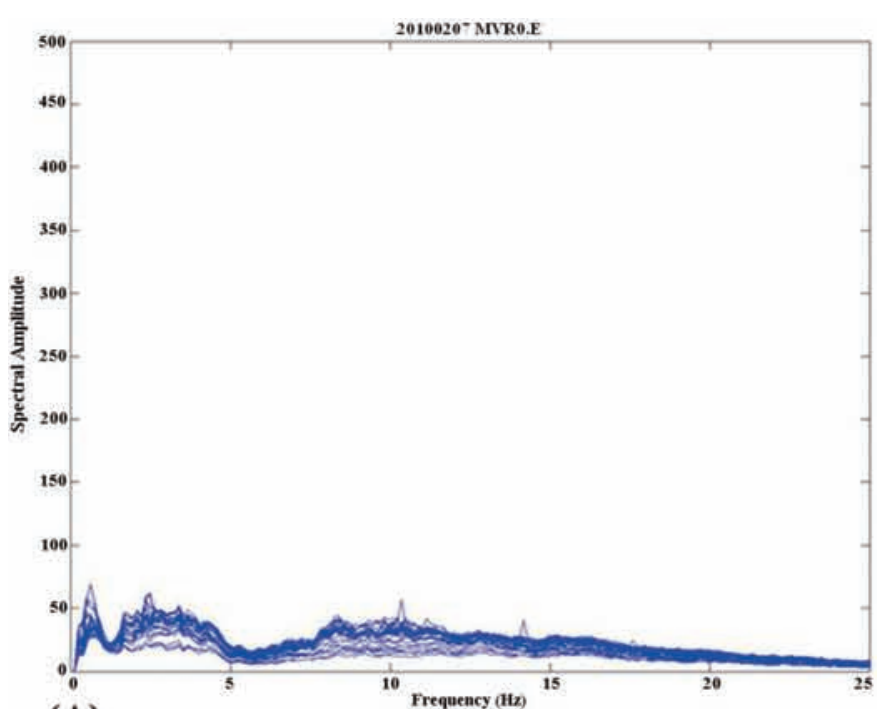

(A)

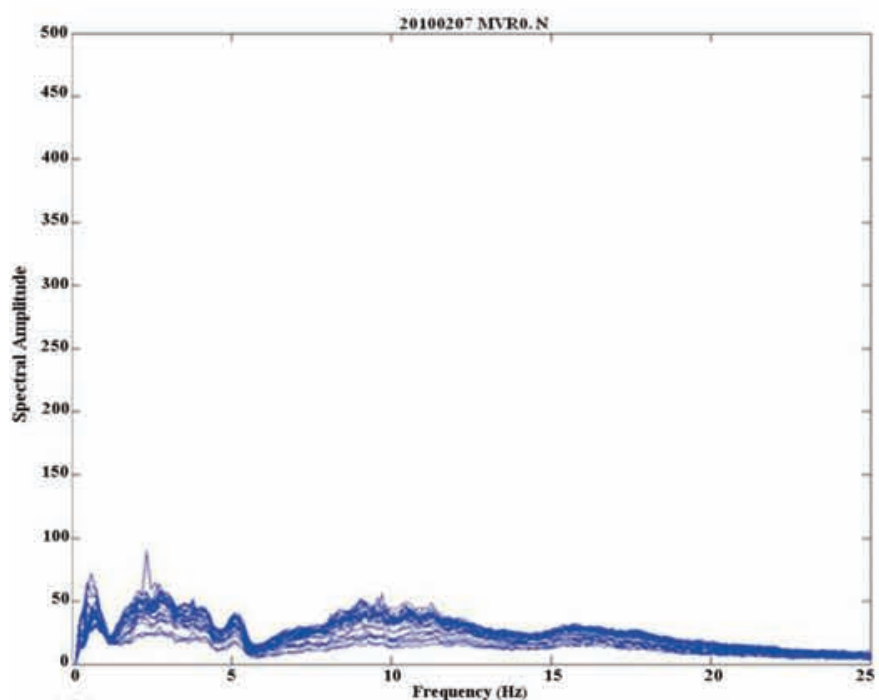

(C)

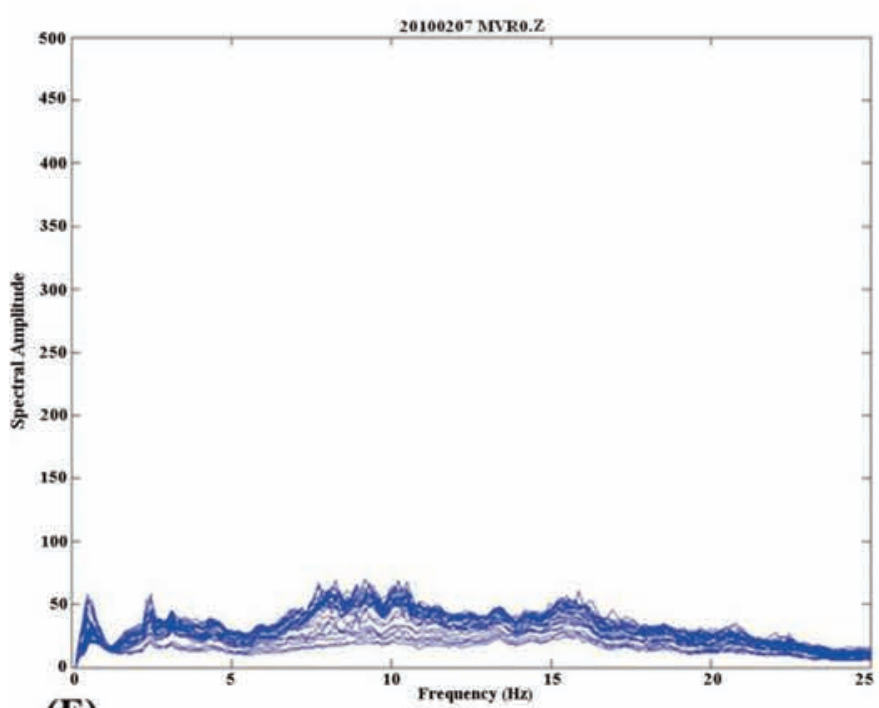

(E)

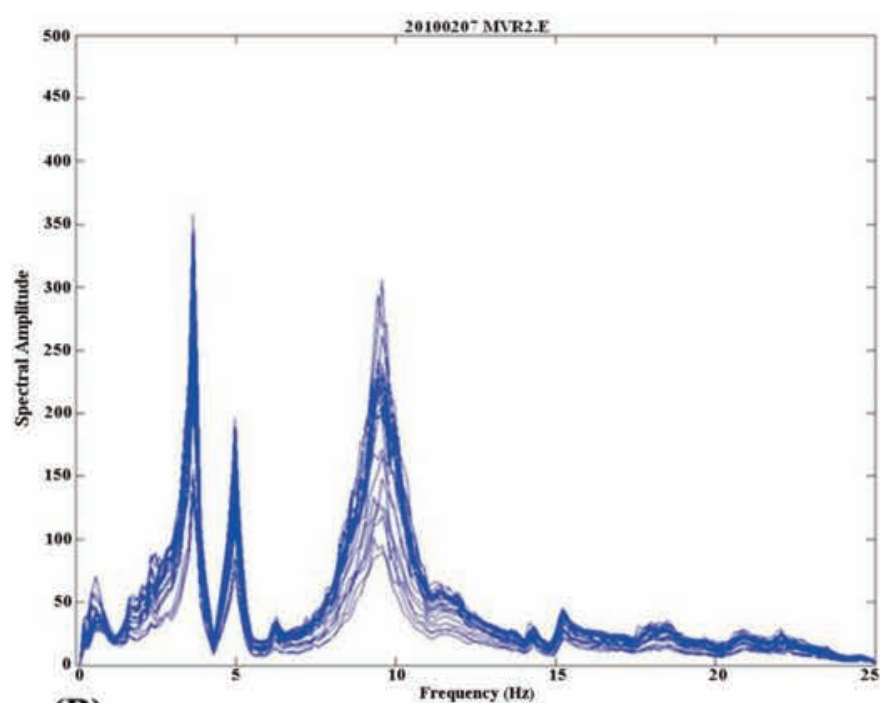

(B)

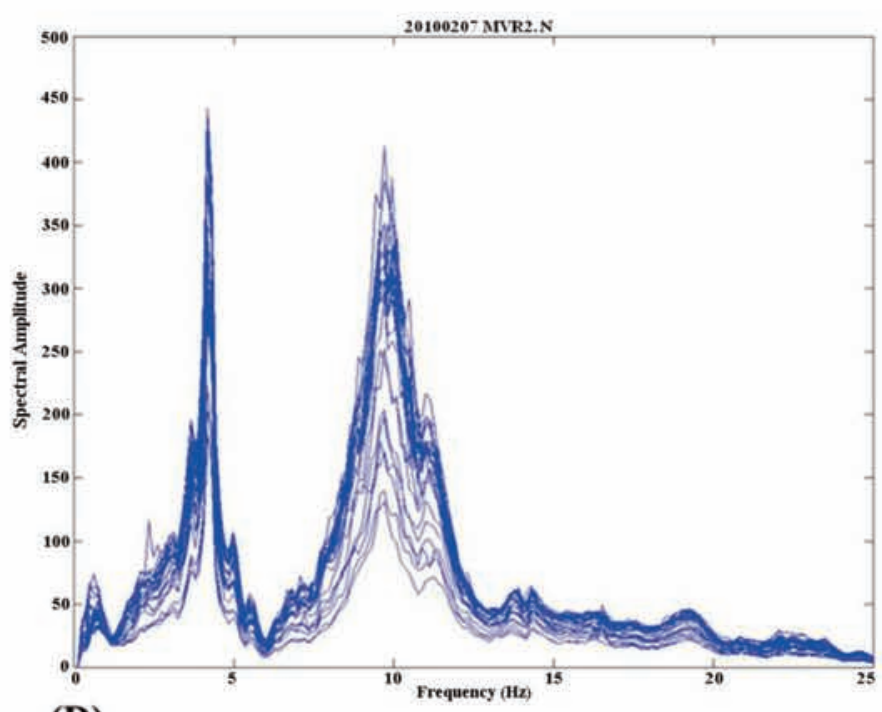

(D)

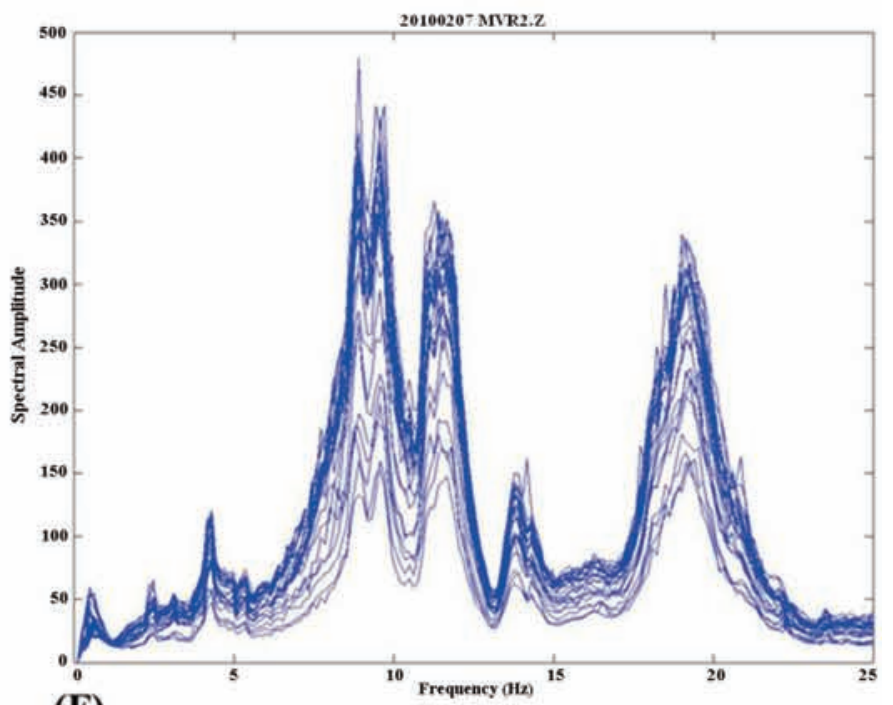

(F)

Figure 3. Spectral amplitudes for the seismic signal recorded by the EW (A), NS (C) and vertical (E) components of station MVR0 (left, as indicated), and for the EW (B), NS (D) and vertical (F) components of station MVR2 (right, as indicated). The signals were recorded on February 7, 2010. Each line corresponds to a 1-h-long time window. 
measurements. The most external room contains the MSR (Figure 1, top left) and the other has the laboratory where the magnetometric data are elaborated (Figure 1, bottom left). The two seismic stations were placed very close each other; MVR2 inside the MSR (Figure 1, top left), in the centre of the floor; and MVR0 outside the MSR, in the laboratory (Figure 1, bottom left), at $5 \mathrm{~m}$ from MVR2. The acquisition started on Friday, February 5, 2010, at 11:00 GMT, and ended on Tuesday, February 9, 2010, at 08:00 GMT.

\section{Data analysis}

The MSR is meant to be isolated from the room it is in, and therefore to strongly attenuate any external influences. If it is built correctly, the spectral amplitudes should be very low across a broad frequency range, as compared to the external spectrum. If this is not the case, the MSR would be transparent to any external motion, and it might even amplify it at some frequencies. Therefore, we investigated

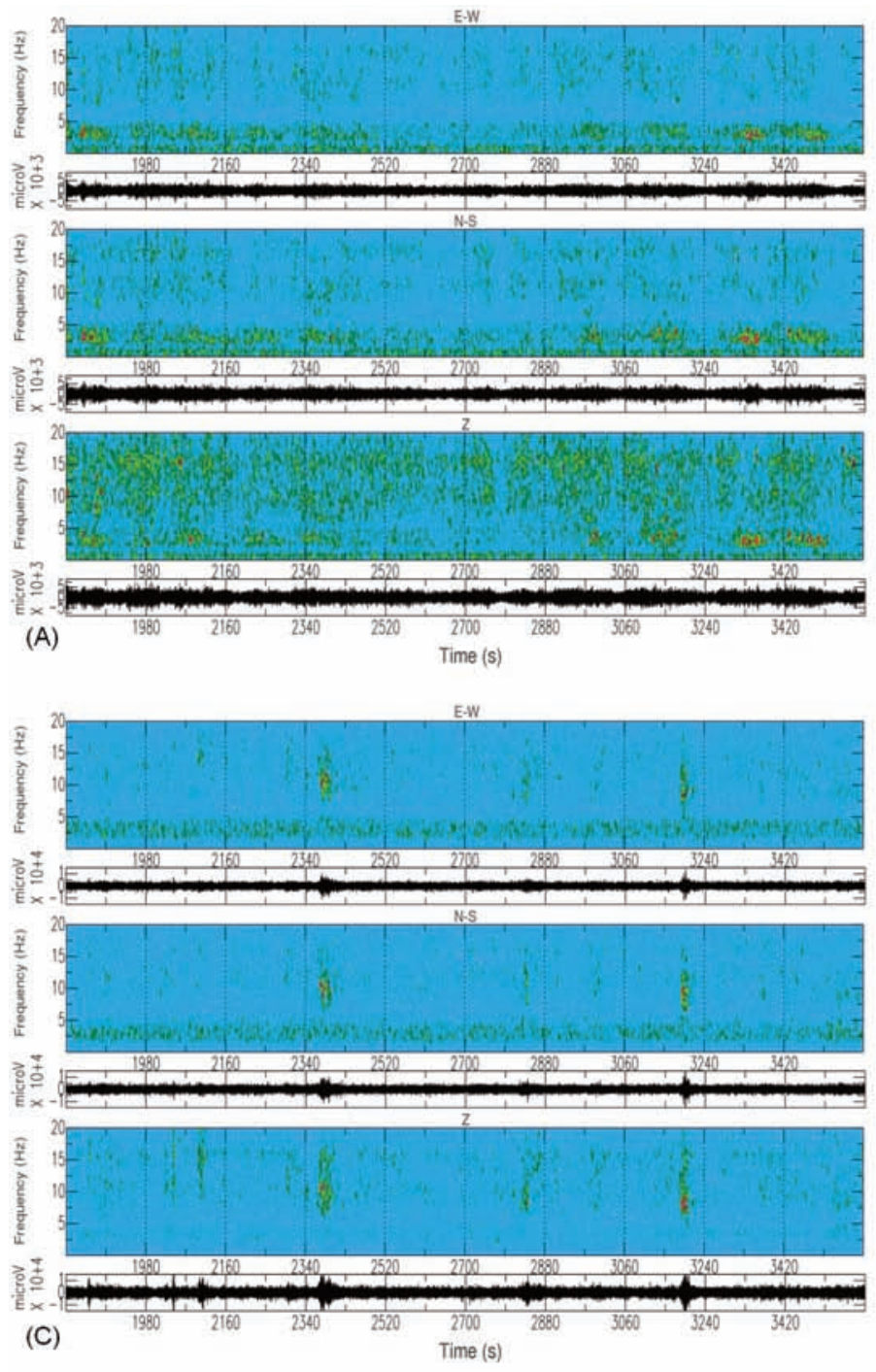

the spectral properties of the weak motion at the stations MVR0 and MVR2.

\subsection{Spectral properties}

The spectral characteristics of the noise recorded at the two stations were inspected by calculating the amplitude of Fourier spectra for 1-h-long time windows. Figure 3 shows the stationary nature of the microtremor, as each spectrum shows the same peaks independent of time. Below $1 \mathrm{~Hz}$, the spectral amplitudes at the two stations are comparable. As a general comment, at frequencies greater than $1 \mathrm{~Hz}$, the amplitude peaks are significantly more energetic for station MVR2. The EW component of MVR2 (Figure 3B) shows the most energetic peaks at $3.6 \mathrm{~Hz}, 5.0$ $\mathrm{Hz}$ and $9.5 \mathrm{~Hz}$, and the NS component at $4.2 \mathrm{~Hz}$ and $9.5 \mathrm{~Hz}$ (Figure 3D). For the vertical component (Figure 3F), there is a double peak in the band from $9 \mathrm{~Hz}$ to $12 \mathrm{~Hz}$ (note the common peak at $9.5 \mathrm{~Hz}$ for all of the three components), a
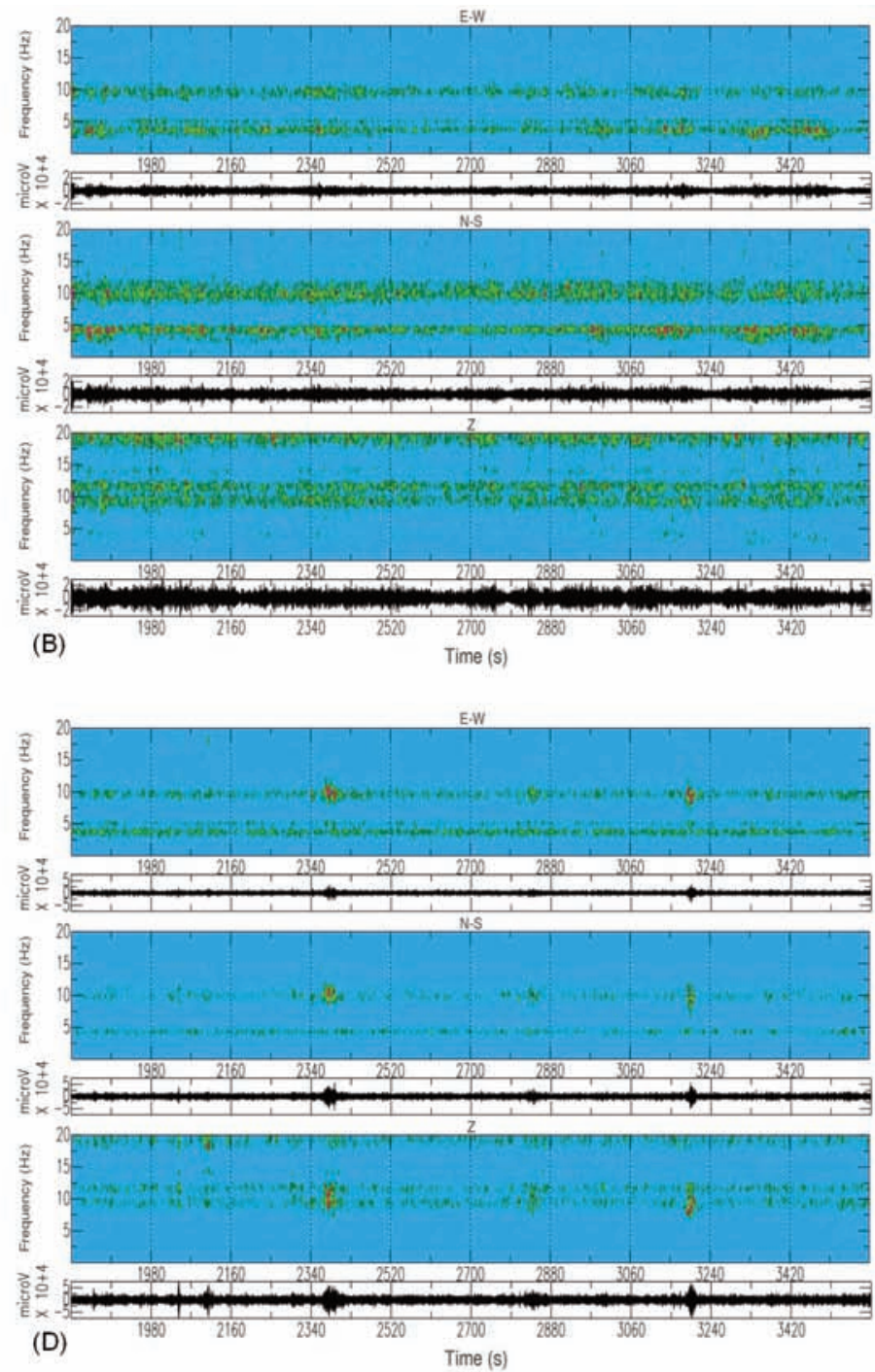

Figure 4. Spectrograms and noise seismograms. Spectral amplitudes (rainbow color scale) as a function of frequency (vertical axes) and time (horizontal axes) with their corresponding seismic signals immediately below each spectrogram, recorded for EW (upper strip), NS (central strip) and Z (bottom strip). (A, C) Station MVR0. (B, D) Station MVR2. (A, B) Recorded on February 7, 2010, between 00:30 and 01:00. (C, D) Recorded on February 7, 2010, between 12:30 and 13:00. 
peak at $14 \mathrm{~Hz}$, and another peak at $19.5 \mathrm{~Hz}$. Moreover, several lower amplitude peaks can be seen in the band from $1 \mathrm{~Hz}$ to $6 \mathrm{~Hz}$. At station MVR0, the spectrum appears mostly flat (Figure 3A, C, E). Most of the energy is concentrated in the band from $1 \mathrm{~Hz}$ to $5 \mathrm{~Hz}$, then around $10 \mathrm{~Hz}$ and $15 \mathrm{~Hz}$, for all of the components.

The persistence of the spectral properties is highlighted in the spectrograms (Figure 4). At station MVR2, the same peaks that are observed in the amplitude spectra (Figure 3B, D, F) appear almost continuously with time (Figure 4B, D). The horizontal components of station MVR0 also show the presence of persistent peaks (Figure $4 \mathrm{~A}, \mathrm{C}$, upper and central strips), while there is a broader frequency content spread over time for the vertical component (Figure 4A, C, bottom strip).

\subsection{Root mean square}

We analyzed the noise characteristics in the time domain through computation of the root mean square (RMS) of the time series, to enhance any common temporal behavior between the two stations. The RMS value was computed on the raw data along 1-h-long time windows (Figure $5 \mathrm{~A}$ ). Moreover, the traces were filtered in two frequency bands: $1 \mathrm{~Hz}$ to $6 \mathrm{~Hz}$, and $5 \mathrm{~Hz}$ to $15 \mathrm{~Hz}$ (Figure $5 \mathrm{~B}$ and $\mathrm{C}$, respectively). We estimated the RMS for the 3-components of the motion (EW, NS and Z).

As can be seen from Figure 5, the RMS shows the daynight cycle at both stations for the raw data and for the 1-6 $\mathrm{Hz}$ and $5-15 \mathrm{~Hz}$ band-filtered signals. These data are typical of anthropogenic activity [Stutzmann et al. 2000, McNamara and Buland 2004]. On Saturday (February 5;2/5) and Sunday (February 6; 2/6) the mean value of the RMS is slightly lower than the other days. This is probably linked to the weekly cycle of the anthropogenic noise. The RMS in the 1-6 Hz band of the vertical component is comparable at both of the stations; in all other cases, the amplitude at MVR2 is significantly larger than at MVR0.

\section{Spectral ratio analyses}

The description of the spectral properties and the RMS behavior above highlights the lack of efficient mechanical isolation of the MSR and the different responses of the two stations to the input wavefield. To investigate the nature of these two responses, we applied two spectral ratio



Figure 5. RMS of the noise amplitudes for the three components of the two seismic stations as functions of time. Red symbols, station MRV0. Blue symbols, station MVR2. A) raw signals. B) 1-6 Hz filtered signals. C) 5-15 Hz filtered signals. Each symbol represents the RMS value calculated over a 1-h-long time window. EW, NS and vertical E components as indicated. 

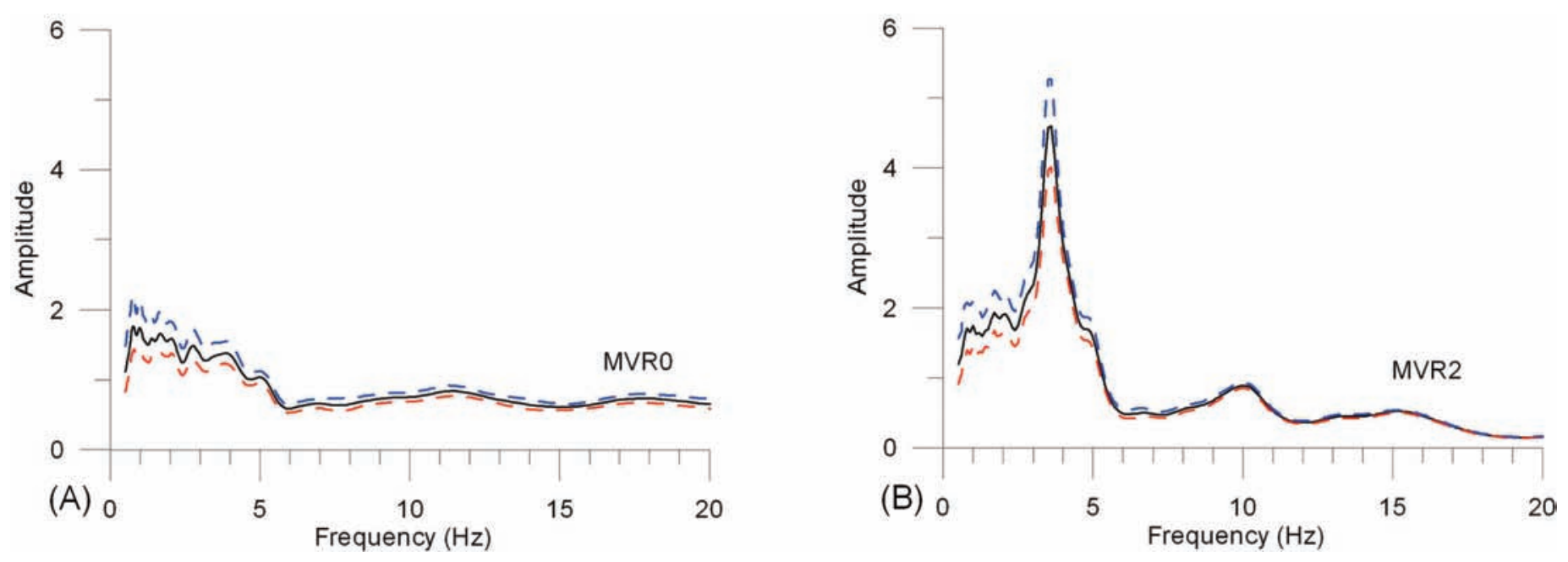

Figure 6. H/V spectral ratio curves for station MVR0 (A) and for station MVR2 (B). Black continuous lines, mean values of the curve. Blue and red dashed lines, standard deviations over the whole period analyzed.

techniques and compared the results, to provide more robustness to the analysis. We started with the horizontal to vertical $(\mathrm{H} / \mathrm{V})$ spectral ratios to retrieve any possible site effects. Keeping in mind the $\mathrm{H} / \mathrm{V}$ results, we then calculated the power spectral density (PSD) ratios with respect to the reference site.

\section{1. $\mathrm{H} / \mathrm{V}$ spectral ratio}

One of the most used techniques based on microtremors is the method of Nakamura [1989, 2000], which consists of the estimation and analysis of the $\mathrm{H} / \mathrm{V}$ spectral ratios of the noise recorded by a single seismic station. Its interpretation has been long debated [Luzon et al. 2001, Malischewsky and Scherbaum 2004], although by following standard criteria for the correct noise acquisition and by checking the conditions under which the results can be considered reliable [Bard and the SESAME Team 2005], the $\mathrm{H} / \mathrm{V}$ curve can be considered to be a good estimation for the transfer function of the S-wave. Moreover, the frequency values at which the peaks occur correctly indicate the resonance frequencies of the site. Many studies have extended this $\mathrm{H} / \mathrm{V}$ method to study interactions between ambient noise and buildings [Gallipoli et al. 2004, Gallipoli et al. 2006, Ditommaso et al. 2009].

The $\mathrm{H} / \mathrm{V}$ ratio is computed as:

$$
s_{i}(f)=\frac{H_{i}(f)}{V_{i}(f)}
$$

where $s_{i}$ is the amplification at the i-th site, $H_{i}$ and $V_{i}$ are, respectively, the horizontal and the vertical component of the spectral amplitude at the i-th site, and $f$ is the frequency.

For each station, the 3-component records were filtered in the $1-20 \mathrm{~Hz}$ band and a short-term average over long-term average (STA/LTA) algorithm was applied to reject transients and disturbances. Then time-windows of $40 \mathrm{~s}$ with an overlap of $30 \%$ were selected. After averaging the EW and
NS components to obtain the horizontal component of the Fourier spectrum, we calculated the spectral ratios between the horizontal and vertical components and we applied the Konno-Omachi smoothing function [Konno and Omachi 1998]. Finally, the arithmetic means of all the H/V ratios over each hourly recording period were calculated. We checked the reliability of the $\mathrm{H} / \mathrm{V}$ curve and the stability conditions for the H/V peak obtained according to the criteria defined in the SESAME project (http://sesame-fp5.obs.ujfgrenoble.fr/SES_TechnicalDoc.htm). The H/V curves for MVR0 and MVR2 are shown in Figure 6 (A and B, respectively). Both of these curves are reliable according to the SESAME criteria, and the peak at $3.6 \mathrm{~Hz}$ for station MVR2 is the only one that satisfies the stability conditions. As a consequence, we can state that the laboratory where station MVR0 was installed is not affected by significant site amplifications. On the contrary, in the MSR, where station MVR2 was installed, there is an anomalous amplification in correspondence wih the resonant frequency $f 0=3.6 \mathrm{~Hz}$. The stability of the $f 0$ value was found all along the recording time (Figure 7). The average amplitude is about 4.4. The errors are from $2 \%$ to $5 \%$ for the resonant frequency, and about $20 \%$ for the amplitude values.

We can conclude that station MVR0 shows a flat $\mathrm{H} / \mathrm{V}$ spectrum, while station MVR2 shows a clear peak at $3.6 \mathrm{~Hz}$. Considering the short distance between the two stations, the anomalous amplification observed for MVR2 is not likely to be a site effect. Moreover, MVR0 is a correct reference site. On these bases, the spectral ratios between the two stations are suitable to retrieve the transfer function of the MSR.

\subsection{Power spectral density}

The PSD curves provide the energy density as a function of the frequency. A PSD curve for ground velocity is computed as the Fourier transform of the autocorrelation function of the noise. For a selected time window with 

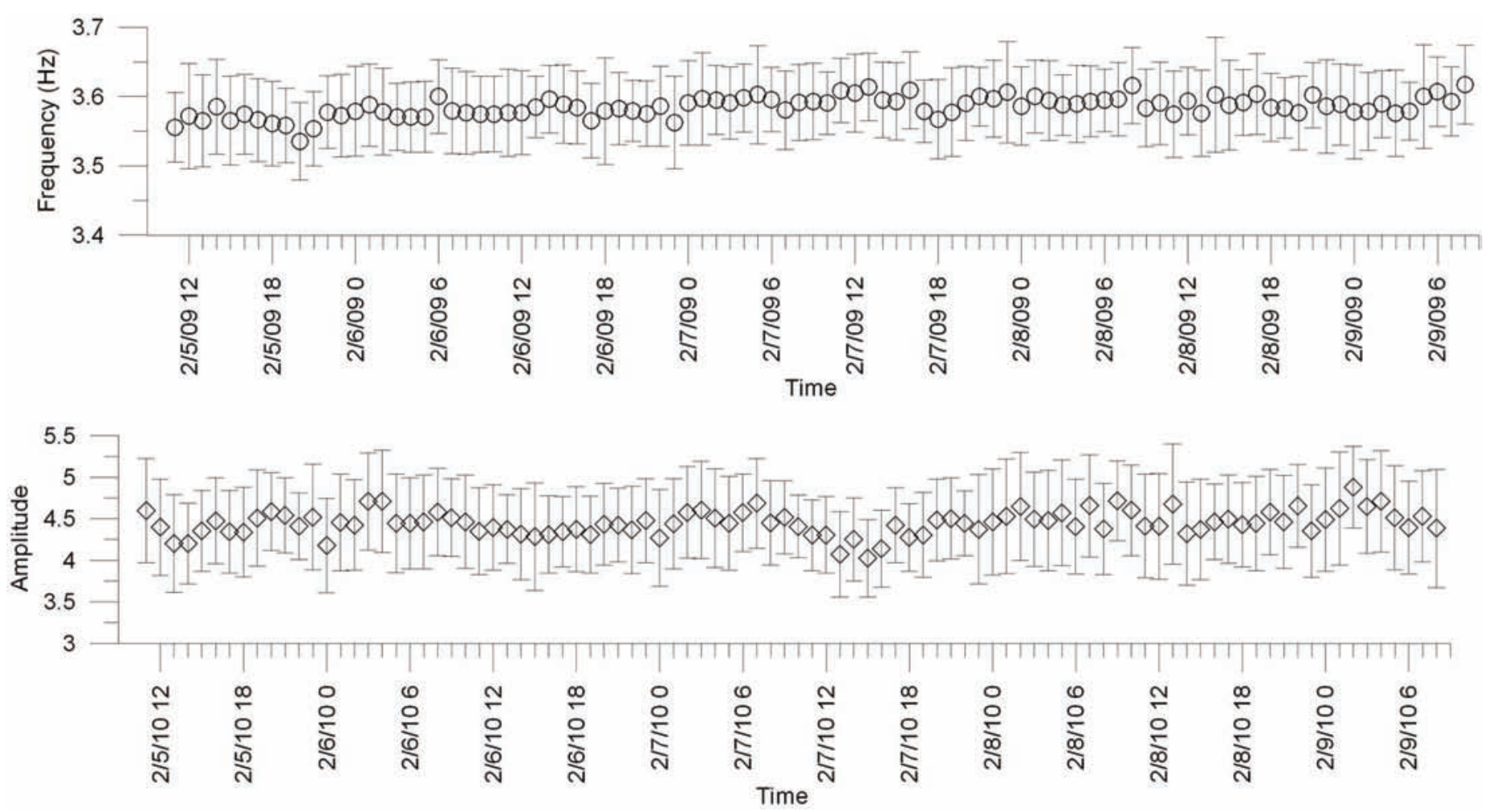

Figure 7. Temporal behaviors of the fundamental frequencies (top) and the corresponding amplitudes (bottom) for station MVR2, with their associated error bars, over 1-h-long time windows.
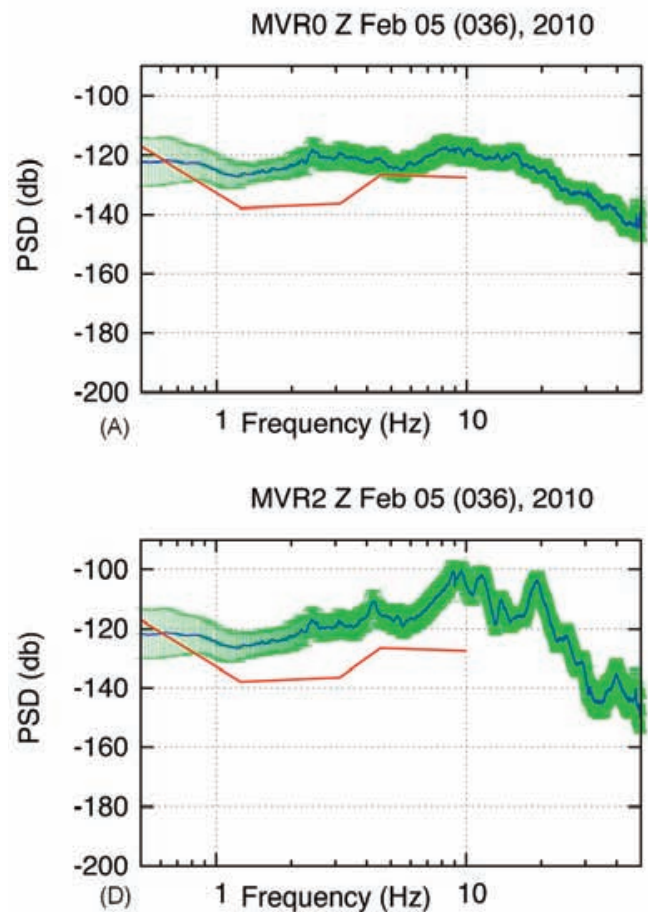

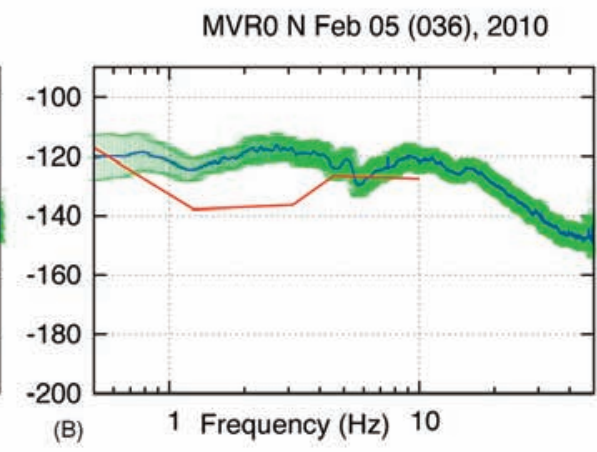

MVR2 N Feb 05 (036), 2010

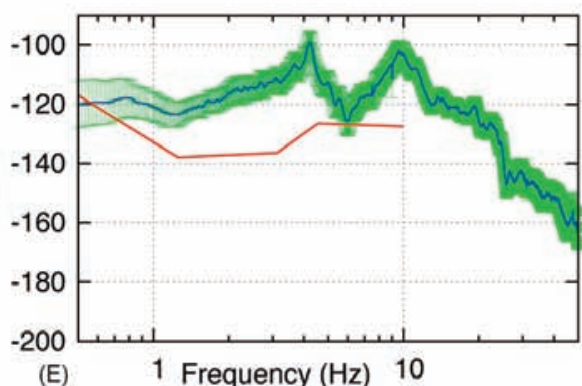

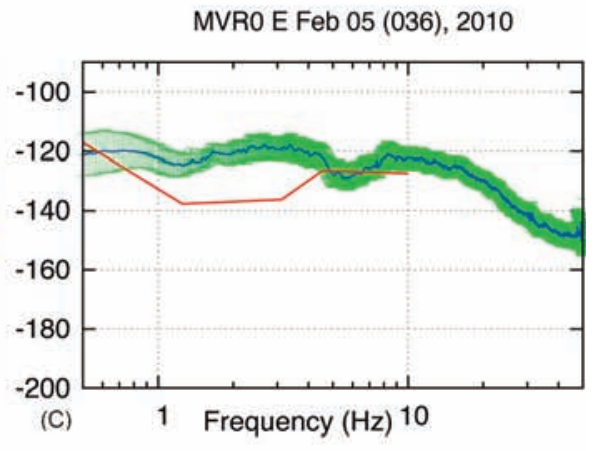

MVR2 E Feb 05 (036), 2010

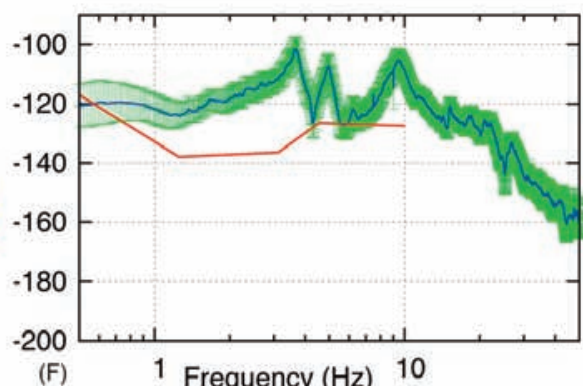

Figure 8. PSD curves for the ground velocity as a function of the frequency for external station MRV0 (A EW, B NS and C vertical component) and internal station MRV2 (D EW, E NS and F vertical component). Red lines, high-level noise Peterson curve, which represents a worldwide reference for the noise. Green bars represent standard deviations of PSD curves. The PSD curves from the external station MVR0 are generally flat, while those from the internal station MVR2 show several peaks in the frequency range of $1 \mathrm{~Hz}$ to $20 \mathrm{~Hz}$, with an increased level of $20 \mathrm{db}$. 

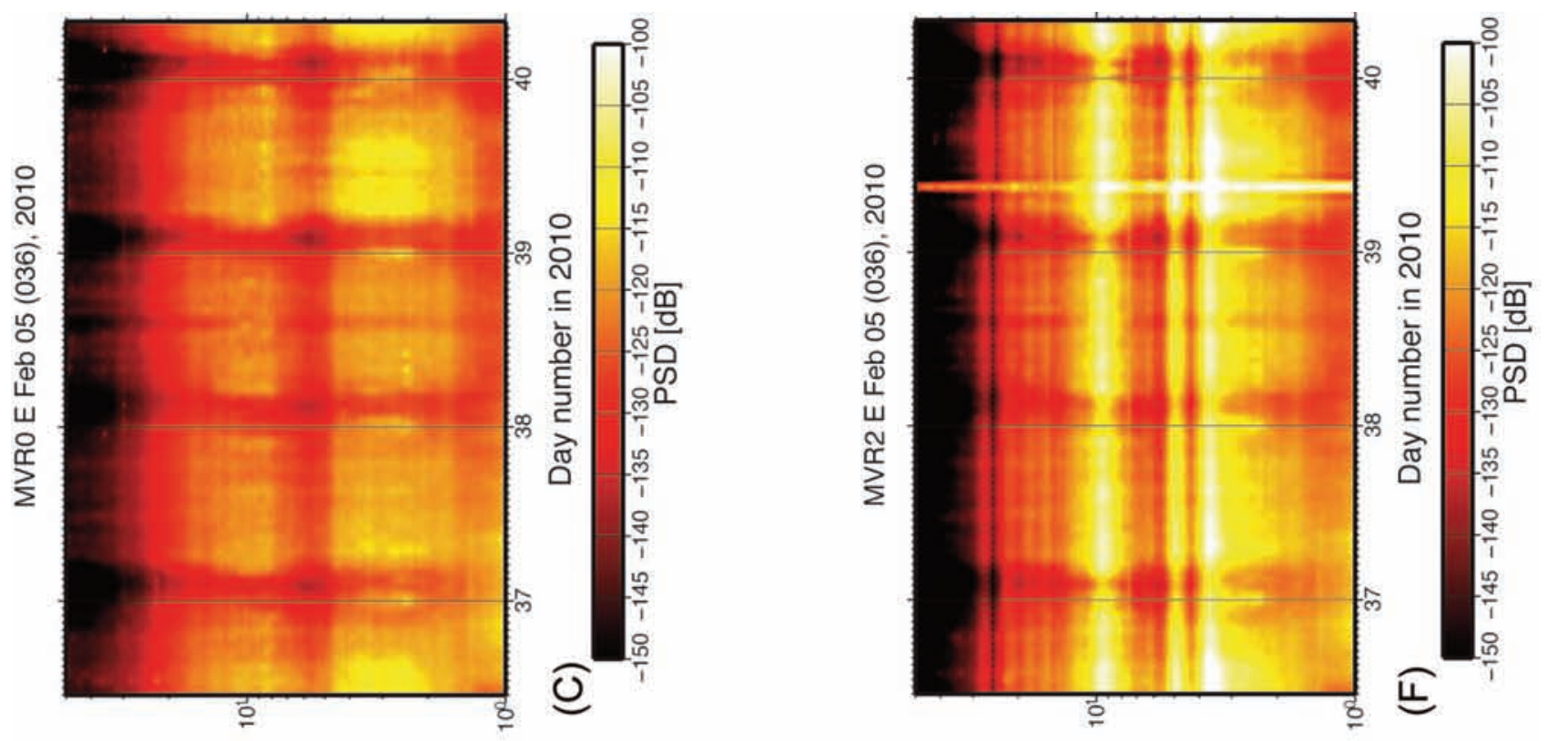

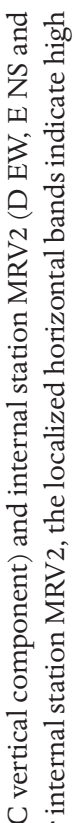
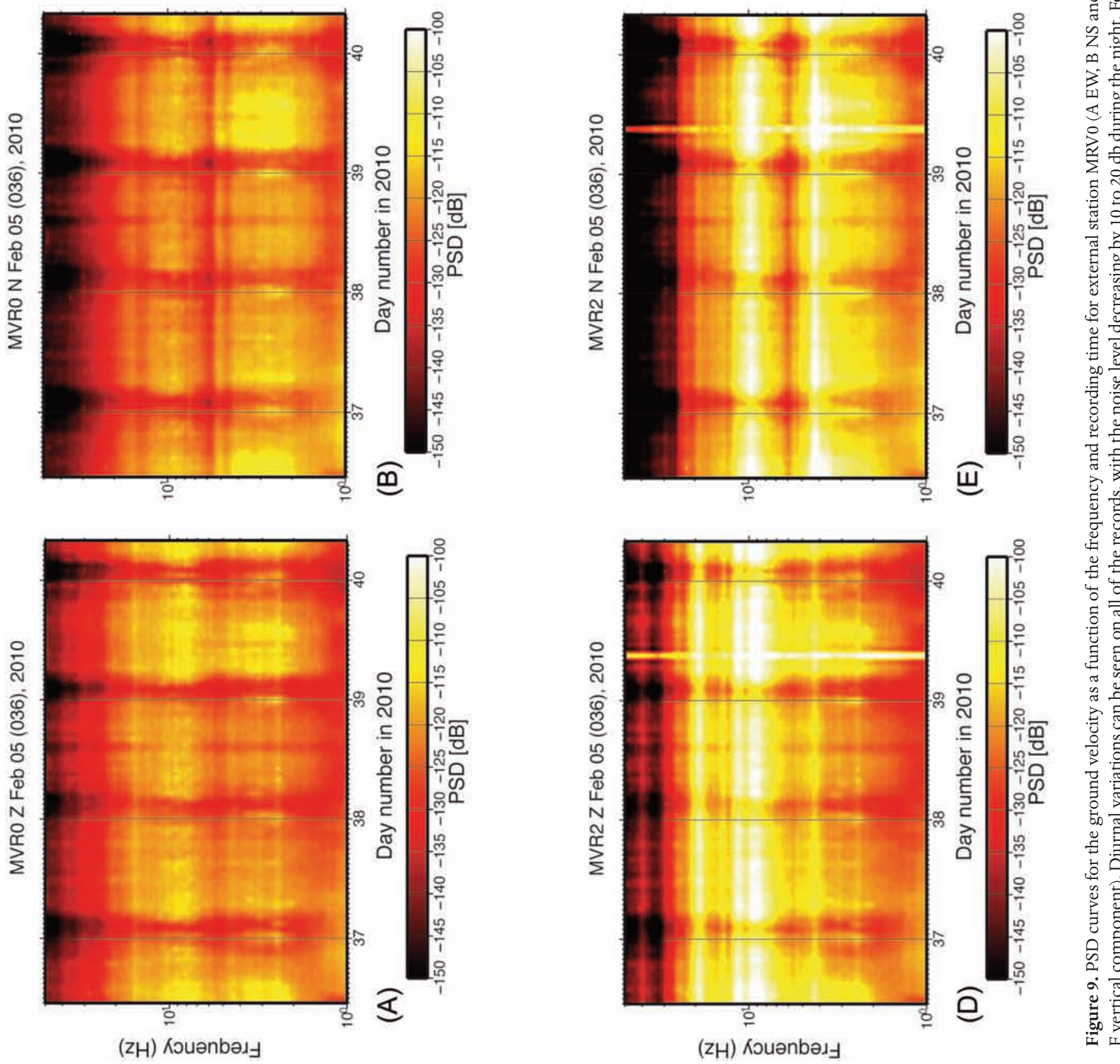

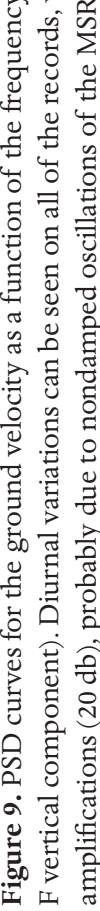


duration $T$, the spectral density curve is given as:

$$
S(\omega)=\frac{F(\omega) F *(\omega)}{T}
$$

where $F(\omega)$ is the Fourier transform of the selected time signal $\mathrm{y}(t)$, and the symbol * (asterisk) denotes the complex conjugation. The PSD is the logarithmic representation of S:

$$
P S D=10 \log S(\omega)
$$

and is measured in decibels.

We computed the PSD curves all along the recording period. In Figure 8, the PSD is shown as functions of the frequency from $0.5 \mathrm{~Hz}$ to $40.0 \mathrm{~Hz}$ for the two stations, MRV0 (Figure 8 A, B and C) and MRV2 (Figure 8 D, E and F). Superimposed, we have also plotted the high-level noise Peterson curve [Peterson 1993] that is obtained by averaging the noise level from a broad number of worldwide seismic stations. This curve represents the reference level according to which we can define the quality of a recording site. The single panels for the two stations are related to the three components (EW, NS and Z) of the motion. Each plot in Figure 8 shows the level of the PSD averaged along the whole recording period with a solid blue line, and the standard deviation at each frequency with green bars. As a first comment, the variation in the noise amplitude is mostly stable with the frequency (about $10 \mathrm{db}$ ), indicating that the changes in the energy are over one order of magnitude during the recording time, independent of the frequency. The curves for the external station MVR0 are approximately flat between $1 \mathrm{~Hz}$ and $20 \mathrm{~Hz}$, with a constant level around $-120 \mathrm{db}$. In addition, the two horizontal components show a small decrease of the PSD level at $6 \mathrm{~Hz}$, with the mean level going down to $-130 \mathrm{db}$. For the internal station MVR2, the PSD curves are more irregular, with several peaks between $1 \mathrm{~Hz}$ and $20 \mathrm{~Hz}$. Some of the peaks are present only in one or two of the components, indicating a complex response of the MSR to these external influences. The EW component shows three prominent peaks at $3.6 \mathrm{~Hz}$ (the same frequency as retrieved from the $\mathrm{H} / \mathrm{V}$ analysis), $5 \mathrm{~Hz}$ and $9.5 \mathrm{~Hz}$, with the corresponding PSD levels ranging between $-100 \mathrm{db}$ and $-105 \mathrm{db}$. For the NS component, there are only two main peaks at $4.2 \mathrm{~Hz}$ and $9.5 \mathrm{~Hz}$, again at $-100 \mathrm{db}$. Finally, for the vertical component, the two peaks at $3.6 \mathrm{~Hz}$ and $5 \mathrm{~Hz}$ are still observable, but they have lower amplitudes. Instead, the most significant peaks here are at higher frequencies: there is a series of peaks between $8 \mathrm{~Hz}$ and $12 \mathrm{~Hz}$, and then another with comparable amplitudes at 19.5 Hz. Figure 9 shows the PSD as a function of time in the same frequency range. Here, in Figure 9, the top panels (A, $B$ and $C$ ) refer to the external station, MVR0, and the bottom panels (D, E and F) to the internal station, MVR2. For both stations, we see a diurnal variation of the PSD, with the noise level dropping down during the night to $10 \mathrm{db}$ to $20 \mathrm{db}$. For the internal station MVR2, at some frequencies we clearly see several localized horizontal bands with strong amplification (on average, $20 \mathrm{db}$ ). Hence the noise level inside the MSR is significantly higher than the external noise at these frequencies all along the recording time. This indicates that the MSR is probably amplifying the external load instead of damping it.

\subsection{PSD spectral ratios}

Absolute values of PSD curves can depend on the actual level of ambient noise, which can show daily variations of one or two orders of magnitude. Although these variations can be followed on both instruments, the evaluation of the amplification of the MSR requires normalization of the amplitude with respect to some reference level. Such normalization is commonly applied for the evaluation of elastic responses of buildings [Mucciarelli et al. 2003, Gallipoli et al. 2006, Ditommaso et al. 2009]. We assumed the reference as the signal recorded at MRV0, the station outside the MSR.
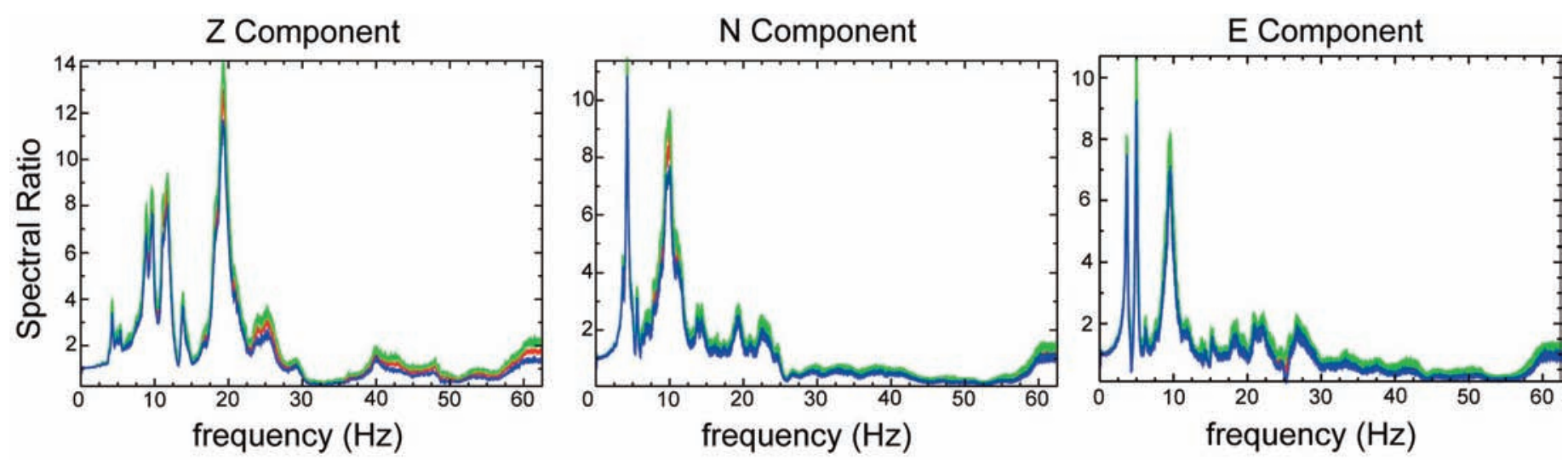

Figure 10. Spectral ratios for each component, between the internal MRV2 and external MRV0 spectra. These relative spectra shows several peaks, with amplitudes between 8 and 12 . 
Since the PSD level of the external station is almost flat, we can assume at the first order that waves at the resonance frequencies that are injected back into the medium by the oscillating MSR do not contaminate the signal at MRV0, and hence that MRV0 reproduces the ambient noise well. Figure 10 shows the PSD spectral ratio component by component as functions of the frequency along a linear scale. We recognize the same peaks that we have already observed in the PSD curves. The spectral ratio for the EW component shows two close peaks at $3.6 \mathrm{~Hz}$ and $5 \mathrm{~Hz}$, with amplitudes of 8 and 10 , respectively. The third peak for the EW component is at 9.5 $\mathrm{Hz}$, with amplitude 8. For the NS component, the largest spectral ratio is at $4.5 \mathrm{~Hz}$, with an amplitude slightly larger than 10. Also, the peak at $9.5 \mathrm{~Hz}$ is present, with an amplitude comparable to that observed for the other horizontal component.

Finally, the spectral ratio for the vertical component shows a different range of resonating frequencies, with three peaks between $8 \mathrm{~Hz}$ and $12 \mathrm{~Hz}$, and the main peak at 19.5 $\mathrm{Hz}$, with an amplitude of 12 . Hence, the shielded room has a complex elastic response. It never dampens the input signal in the $1 \mathrm{~Hz}$ to $20 \mathrm{~Hz}$ range. Indeed the MSR amplifies the external motion at some frequencies up to one order of magnitude, with the resonance frequencies dependent on the specific direction of the wavefield.

\section{Conclusions}

The application of several analysis techniques to the background noise recorded by these stations external and internal to the MSR allowed us to establish the nature of the anomalous peaks retrieved in the magnetic field flux spectra (Figure 2) of the SQUID device.

The first useful piece of information comes from the RMS analysis. The presence of the day-night cycles (Figures 5) shows the anthropogenic origin of the signals for both the internal and external stations. A second important observation comes from the spectral analysis. Inside the MSR, the energy carried by the seismic noise wavefield is significant higher than that outside the MSR, all along the recording time, although the energy is distributed over the same frequency bands. This evidence is confirmed by the PSD analysis (Figures 8 and 9). The most significant results are obtained with the H/V (Figures 6 and 7) and the PSD spectral ratios for the reference site (Figure 10). In the first case, we found an almost flat spectrum outside the MSR, while the H/V curve inside the MSR shows a clear peak at $3.6 \mathrm{~Hz}$, which corresponds to one of the most energetic anomalous peaks retrieved in the magnetic flux spectra (Figure 2). This means that the amplification retrieved inside the MSR cannot be attributed to a site effect, and that station MVR0 can be considered a good reference site. The internal over external spectral ratios show a variety of peaks, some of which are present only on one or two components, which reflects a complex response of the MSR to these external vibrations.

All of the techniques applied yield the same conclusions: the input signal of anthropogenic origin is not damped by the MSR in the frequency range of $1 \mathrm{~Hz}$ to 20 $\mathrm{Hz}$. It is instead amplified at some frequencies, by up to one order of magnitude, with the resonance frequencies being dependent on the specific direction of the external motion. This is thus evidence of the lack of mechanical isolation of the MSR that hosts this MEG system, and it might also explain a phenomenon observed in the course of SQUID operation. Indeed, the MSR is equipped with a stirrup that couples it to the soil, and it was noted that the anomalous peaks in the magnetic flux spectrum are more energetic when the stirrup is disconnected. We are thus confident that the problem of these spurious peaks can be resolved through reconsideration of the criteria used to isolate the MSR from the mechanical input.

Finally, we want to highlight the efficiency of these techniques based on the background noise in the prompt identification of structural defects. The low cost and discretion of the experiment, the immediate availability of the data, and the robustness of the results reveal how ambient noise methods can offer concrete benefits for structural analysis. Considering all of these advantages, these tests might also be performed before the building of such a structure, to extract the main features of the seismic noise at the investigating site, and thus to define the attenuation mechanisms which will reduce the ambient noise to the acceptance level.

Acknowledgements. This study was realized thanks to the contributions of Dr. Francesca Bianco, Istituto Nazionale di Geofisica e Vulcanologia, Prof. Aldo Zollo, «Federico II» University of Naples, and the Mobile Seismic Network of INGV-Osservatorio Vesuviano. We are grateful to Dr. Maurizio Vassallo, from AMRA Scarl, for helpful discussions. Dr. Laura Bagnasco, Politecnico of Torino, is fully acknowledged for her help in the installation of the seismic stations. We thank the Villa Russo Administration for having hosted the experiment. Finally, we are grateful to two anonymous reviewers whose suggestions helped us to improve the manuscript.

\section{References}

Bard, P.-Y. and the SESAME Team (2005). Guidelines for the implementation of the $\mathrm{H} / \mathrm{V}$ spectral ratio technique on ambient vibrations - measurements, processing and interpretations, SESAME European Research Project EVG1-CT-2000-00026 D23.12; available online at http:// sesame-fp5.obs.ujf-grenoble.fr.

Cohen, D. (1968). Magnetoencephalography: evidence of magnetic fields produced by alpha-rhythm currents, Science, 161, 784; doi: 10.1126/science.161.3843.784.

Cohen, D. (1972). Magnetoencephalography: detection of the brain's electrical activity with a superconducting magnetometer, Science, 175, 664; doi: 10.1126/science.175. 4022.664 . 
Ditommaso, R., S. Parolai, M. Mucciarelli, S. Eggert, M. Sobiesiak and J. Zschau (2009). Monitoring the response and the back-radiated energy of a building subjected to ambient vibration and impulsive action: the Falkenhof Tower (Potsdam, Germany), Bull. Earthq. Eng.; doi: 10.1007/s10518-009-9151-4.

Gallipoli, M.R., M. Mucciarelli, R.R. Castro, G. Monachesi and P. Contri (2004). Structure, soil-structure response and effects of damage based on observations of horizontalto-vertical spectral ratios of microtremors, Soil Dyn. Earthq. Eng., 24, 487-495.

Gallipoli, M.R., M. Mucciarelli, F. Ponzo, M. Dolce, E. D'Alema and M. Maistrello (2006). Buildings as a seismic source: analysis of a release test at Bagnoli, Italy, B. Seismol. Soc. Am, 96, 2457-2464; doi: 10.1785/0120060015.

Group of Scientific Experts (1995). Conference Room Paper 243, Conference on Disarment, United Nations, Geneva, Switzerland.

Hamalainen, R., R.J. Ilmoniemi, J. Knuutila and O.V. Loounasmaa (1993). Magnetoencephalography - theory, instrumentation, and applications to noninvasive studies of the working human brain, Rev. Mod. Phys., 65, 413-497.

Konno, K. and T. Ohmachi (1998). Ground-motion characteristics estimated from spectral ratios between horizontal and vertical components of microtremors, B. Seismol. Soc. Am., 88, 228-241.

Luzon, F., Z. Al Yuncha, F.J. Sanchez-Sesma and C. OrtizAleman (2001). A numerical experiment on the horizontal to vertical spectral ratio in flat sedimentary basins, Pure Appl. Geophys., 158, 2451-2461.

Malischewsky, P.G. and F. Scherbaum (2004). Love's formula and $\mathrm{H} / \mathrm{V}$-ratios (ellipticity) of Rayleigh waves, Wave Motion, 40, 57-67.

McNamara, D.E. and R.P. Buland (2004). Ambient noise levels in the continental United States, B. Seismol. Soc. Am., 94, 1517-1527.

Mucciarelli, M., M.R. Gallipoli, F. Ponzo, M. Dolce (2003). Seismic waves generated by oscillating buildings: analysis of a release test, Soil Dynam. Earthq. Eng., 23, 255-262.

Nakamura, Y. (1989). A Method for Dynamic Characteristics Estimation of Subsurface using Microtremors on the Ground Surface, Quarterly Report of Railway Technical Research Institute (RTRI), 30 (1).

Nakamura, Y. (2000). Clear identification of fundamental idea of Nakamura's technique and its applications, In: Proc. 12th World Conference on the Earthquake Engineering, Auckland, New Zealand.

Peterson, J. (1993). Observations and modeling of background seismic noise, US Geol. Surv. Open-File Rept. 93.

Rombetto, S. (2009). Sistema multisensoriale a 163 canali per magnetoencefalografia, CNR-IC Internal Report 171/ 09.

Stutzmann, E., G, Roult and L. Astiz (2000). GEOSCOPE station noise levels, B. Seismol. Soc. Am., 90, 690-701.
Williamson, S.J. and L. Kaufman (1990). Auditory Evoked Magnetic Fields and Electric Potentials. S. Karger, Basel.

${ }^{\star}$ Corresponding author: Paola Cusano,

Istituto Nazionale di Geofisica e Vulcanologia, sezione di Napoli, Osservatorio Vesuviano, Naples, Italy; email: paola.cusano@ov.ingv.it.

(C) 2011 by the Istituto Nazionale di Geofisica e Vulcanologia. All rights reserved. 